September 2020

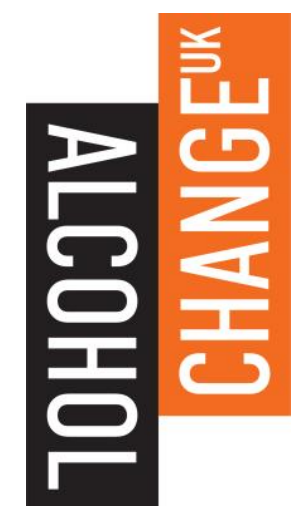

\title{
Alcohol and drug use disorders in patients with cancer and caregivers: effects on caregiver burden
}

Dr Katherine Webber, Dr Andrew N Davies, Dr Charlotte Leach, Dr Anna Bradley 


\section{Author details}

Dr Katherine Webber, Department of Palliative Medicine, Royal Surrey County Hospital Dr Andrew N Davies, Department of Palliative Medicine, Royal Surrey County Hospital Dr Charlotte Leach, Department of Palliative Medicine, Royal Surrey County Hospital Dr Anna Bradley, Department of Palliative Medicine, Royal Surrey County Hospital

\section{Contact person details}

Dr Katherine Webber, Department of Palliative Medicine Royal Surrey County Hospital, Guildford, Surrey, GU2 7XX

Email: kwebber1@nhs.net

\section{Acknowledgements}

This study was funded by Alcohol Change UK and supported by the National Institute of Health Research. We would like to thank all investigators and recruiters at our sites.

This report was funded by Alcohol Change UK. Alcohol Change UK works to significantly reduce serious alcohol harm in the UK. We create evidence-driven change by working towards five key changes: improved knowledge, better policies and regulation, shifted cultural norms, improved drinking behaviours, and more and better support and treatment.

Find out more at alcoholchange.org.uk.

Opinions and recommendations expressed in this report are those of the authors. 


\section{Contents}

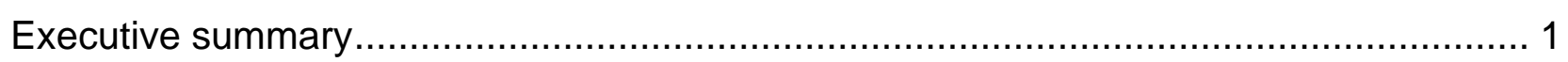

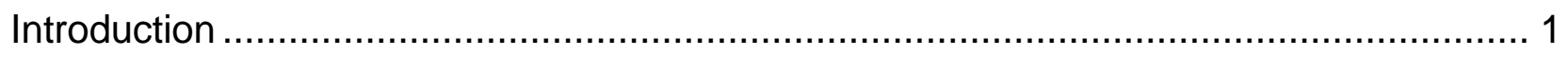

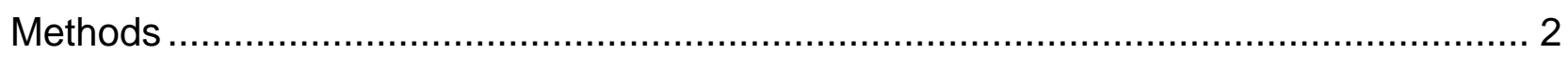

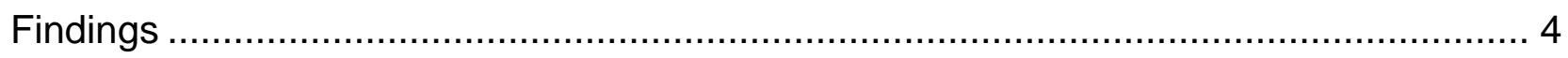

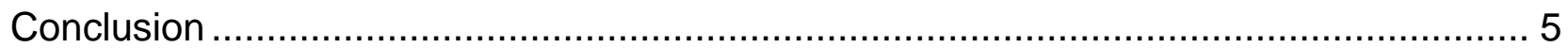

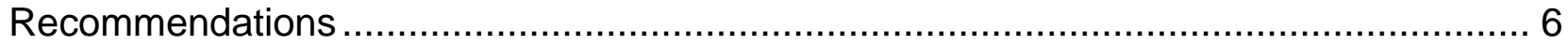

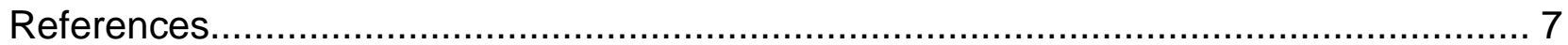





\section{Executive summary}

- $5 \%$ of cancer patients and $8 \%$ of caregivers screened positively for alcohol use disorders on the AUDIT.

- $6 \%$ of patients and $3 \%$ of caregivers screened positively for drug abuse on the DAST10.

- Caregivers screening positively for drug and alcohol problems had significantly higher carer burden scores.

- Carer burden was not affected by the patient's drug or alcohol abuse issues.

\section{Introduction}

More than 300000 people are diagnosed with cancer annually in the United Kingdom. Half of those diagnosed will not survive the disease and are living with advanced cancer for a period of time (Cancer Research UK 2016). It is estimated that $8 \%$ of cancer deaths in men, and $3 \%$ in women, are attributable to excessive alcohol consumption (Schultz et al 2011, World Health Organisation 2007). However, it is less well known whether excess alcohol consumption continues to be, or starts to be, a problem during the course of the illness. Living with cancer, having burdensome treatments and facing the end of life have a significant emotional impact. It is well documented that rates of psychological illness are higher in people with cancer than the general population (Hotopf et al 2002, McManus et al 2007). However, it is unclear whether alcohol and drug use disorders coexist with these problems.

There have been a number of retrospective studies assessing the prevalence of alcohol dependence in advanced cancer patients in North America and Europe (Parsons et al 2008, Jenkins et al 1998, Jenkins et al 2000, Bruera et al 1995, Bruera et al 2000, Mercadenate et al 2015). This is reported as being 4-38\% using the CAGE questionnaire and $18 \%$ using the Alcohol Use Disorders Identification Tool (AUDIT) (Webber and Davie 2012). These results are difficult to interpret as the CAGE refers to lifetime use, not recent use, the datasets were small and retrospective and a number of included patients had missing data.

Many people with advanced cancer are appropriately prescribed strong painkillers for their problems. However, there is limited published United Kingdom data assessing whether drug abuse exists in this population, either from prescribed opioids or other drugs. It is an important distinction to make in such patients to enable safe prescribing and enhancing quality of life. In the United States prevalence rates of drug abuse in cancer patients range from 3-19\% however population level issues are considerably higher in the U.S. making it difficult to extrapolate (Derogatis et al 1983, Webber and Haber 2008, Yennurajalingam et al 2018). 
Caregivers of advanced cancer patients suffer higher levels of distress and lower levels of well-being as compared to age-matched non-caregivers (Pinquart and Sorenson 2003, Grov et al 2005). Caregivers of patients with other chronic problems (head injury, dementia) have high levels of alcohol use disorders and these are related to worse health outcomes and coping (Bayen et al 2014, Gallant et al 1997). A prospective study from Europe assessed cancer caregivers using the CAGE questionnaire; eleven percent screened positively for alcohol dependence (Rumpold et al 2015)). They did not specifically assess the relationship between alcohol dependence and other psychiatric problems or caregiver burden and there was no assessment of drug abuse problems.

There are few published studies that primarily assess the prevalence of alcohol and drug use disorders in both cancer patients and their caregivers. There are limited datasets evaluating the relationship between alcohol and drug abuse and carer burden, as well as the impact of the cancer patient's behaviours and symptoms on carer burden.

The aim of this study, supported by Alcohol Change UK, was to establish the prevalence of alcohol and drug use disorders in a large cohort of advanced cancer patients and their caregivers. Secondary aims were to evaluate the relationship between these problems and symptoms and carer burden. An overarching purpose of the study is to establish a large accurate dataset to determine if screening would be required for certain cohorts.

\section{Methods}

This study recruited cancer patients across eleven United Kingdom sites. Five centres were National Health Service Hospitals and six sites were independent hospices.

Patients were recruited from hospital inpatient and outpatient departments, and hospice inpatient units and community services. Patients aged $>18$ years with a diagnosis of advanced cancer and their primary caregiver, as identified by the patient, were approached. Patients without a caregiver were excluded.

This study has U.K. Research Ethics Committee approval (IRAS 198753). All participants provided written informed consent.

\section{Sample characteristics}

Patient and caregiver demographic data were collected at interview. Details about prescribed painkillers and diagnosis were gathered by interview and also chart review. Smoking status was obtained for both patients and their caregivers.

\section{Assessment measures}

The patient completed the following measures: 
- Alcohol Use Disorders Identification Test. A validated questionnaire to screen for the presence of alcohol misuse in the last 12 months. A cut off score of $\geq 8$ was used to categorise alcohol misuse (Babor et al 2001).

- CAGE questionnaire (Mayfield, McLeod and Hall 1974). Four questions about lifetime alcohol consumption. A cut off score of 2 or more positive answers was used to determine alcohol dependence.

- Memorial Symptom Assessment Scale-Short Form (MSAS-SF). A 32-item validated questionnaire assessing physical and psychological symptoms. (Chang et al 2000)

- The data will be presented using the three subscales, global distress index (GDI), physical subscale (PHYS) and psychological subscale (PSYCH). PSYCH has been validated to detect anxiety and depressive disorders (Webber and Davies 2011)

- Drug abuse screening test (DAST 10). A validated ten item questionnaire to assess for drugs abuse over the previous 12 months (Yudko et al 2007). A cut-off score of $\geq 2$ was used to determine drug abuse.

The caregiver completed the following:

- Alcohol Use Disorders Identification Test.

- CAGE questionnaire

- Drug abuse screening test (DAST-10).

- Zarit burden inventory (ZBI). A validated 29 item scale designed to measure feelings of burden experienced by caregivers of medically ill persons. A cut off score of $\geq 21$ was used to determine significant carer burden. (Zarit, Reever and Back-Peterson 1980)

\section{Statistical analysis}

A sample size of 500 patients was calculated to estimate the prevalence of alcohol use disorders according to the AUDIT with a two-sided 95\% confidence interval; according to expected proportion $18 \%+/-3.3 \%$ ).

Patient and caregivers were categorised into cases and non-cases of alcohol use disorders (AUDIT scale), alcohol dependence (CAGE) and drug abuse (DAST-10) to determine the prevalence of each. These different groups were compared with continuous variable data to establish the relationship between alcohol and drug misuse and the patient's physical and psychological symptoms (MSAS-SF) and carer burden (Zarit). A comparison was also made between the patient's symptom scales and carer burden. Comparisons between categorical and continuous variables was made using the t-test. Data was analysed using SPSS software. 


\section{Findings}

\section{Patient and caregiver characteristics}

A total of five hundred and seven patients and their caregivers were recruited over eleven United Kingdom sites (744 approached, 237 declined to enter). Forty-eight percent were recruited from hospital sites and fifty two percent from hospices.

The median age of patients was 68 and their caregivers was 66 . Fifty four percent of patients were male. The majority of caregivers were spouses or partners. Two hundred and thirteen carers (42\%) experienced significant carer burden as defined by the Zarit Burden Inventory (ZBI).

\section{Patient and caregiver prevalence rates of alcohol and drug misuse}

Twenty-seven patients (5\%) and forty-four caregivers (8\%) screened positively for alcohol use disorders on the AUDIT. In eight cases both the patient and caregiver screened positively.

Fifty-seven patients (11\%) and forty-eight caregivers (9\%) screened positively for lifetime alcohol dependence using the CAGE questionnaire.

Eighty five percent $(85 \%)$ of patients, and twenty seven percent $(27 \%)$ of carers, were taking regular painkillers. The majority of painkillers were prescribed opioids. Thirty patients $(6 \%)$, and sixteen caregivers (3\%), screened positively for drug abuse problems on the DAST-10.

\section{Factors associated with a high carer burden}

There was a significantly higher caregiver burden score in caregivers screening positively for alcohol and drug abuse problems on the AUDIT, CAGE and DAST-10.

A higher burden was also experienced by caregivers if patients had worse physical and psychological symptoms.

Caregiver burden was not affected by the patient's drug or alcohol abuse issues.

\section{Relationship between alcohol and drug abuse and patient's symptoms}

Patients with AUDIT defined alcohol problems had lower global distress index (MSASGDI) and physical symptom scores (MSAS-PHYS) than those without. There was no difference in psychological symptoms (MSAS-PSYCH) between groups.

There was no difference in physical or psychological symptoms between patients screening positively and negatively with the CAGE or DAST-10. 


\section{Conclusion}

The prevalence of cancer patients' alcohol use disorders and dependence was lower than reported in previous studies. Only $5 \%$ of patients had harmful levels of alcohol use disorders in the previous year (AUDIT positive) with a higher number having a positive CAGE (11\%). The CAGE questionnaire assessed lifetime dependence rather than current issues suggesting a proportion of patients had previously had problems but weren't currently using alcohol harmfully (Mayfield, McLeod and Hall 1974).

The level of current alcohol use disorders in patients and caregivers is lower than the general population for this age range taking into account sex differences (NHS Digital Survey 2014). However, lifetime alcohol dependence rates (CAGE) are higher than equivalent general population for both patients and caregivers (NHS Digital Survey 2014). This suggests that individuals with previous issues do not necessarily have current patterns of misuse but must be evaluated carefully and non-judgementally.

Eighty-five percent of patients were taking prescribed painkillers and six percent screened positively for drug abuse. This is the first estimated prevalence rate of drug abuse in cancer patients in the United Kingdom and is higher than general population levels accounting for age (aged 55+ drug abuse rates are less than 1\%) (NHS Digital Survey 2014). This indicates that drug abuse is an issue that should be assessed when prescribing painkillers for all cancer patients.

Studies from the United States report a prevalence rate of 3-19\% for drug abuse in cancer patients however this coincides with a much higher general population rate for the same age group (Derogatis et al 1983, Webber and Haber 2008 and Yennurajalingam et al 2018).

Twenty-seven percent of caregivers were taking prescribed painkillers. This is higher than United Kingdom general population data and indicates that caregivers have significant health care needs and morbidity of their own (Office of National Statistics 2018). Whilst this study did not look in detail at this issue it supports published data about the physical and psychological health problems prevalent in caregivers (Office of National Statistics 2018). Three percent of caregivers screened positively for drug abuse problems which is higher than general population rates for this age group (NHS Digital Survey 2014). In one-third of cases both patient and caregiver screened positively suggesting this is an issue that must be assessed in both cases. There is no published analogous data for the caregivers of cancer patients and it raises important issues about prescribing and screening of this group. Concurrent alcohol and drug abuse problems co-existed in only three patients and three caregivers.

There was a significantly higher carer burden score associated with caregiver alcohol and drug use disorders and lifetime alcohol dependence. It is not known whether this is cause or effect. A previous European study did not identify a positive CAGE questionnaire as a risk factor for carer burden (Rumpold et al 2015). 
A higher carer burden was also associated with patient's physical and psychological symptom burden. The causes of caregiver burden are complex but the literature reports a stronger association between burden and patient's physical care needs, social networks and information needs than symptoms (Hsu et al 2017,Hsu et al 2014). There has been no previous reported association between Zarit burden score and symptoms reported on the MSAS-SF. This is an important finding that warrants further investigation.

\section{Study limitations}

There were limitations to this study. Firstly, the methods used to define alcohol and drug use disorders and lifetime alcohol dependence were screening tools. They are indicative of problems but a structured clinical interview would be used in practice to diagnose these issues. Secondly, the prevalence rates were low and the study was not powered to detect differences between groups. Despite this the fact that differences were seen reveals a powerful link between data described above. There were a considerable number of people who declined to take part in the study. This was predominantly due to fatigue and caregiver not being present during working hours. However, it is possible that people with alcohol and drug use disorders were more reluctant to take part thus underestimating the prevalence. Lastly, the study took place in the United Kingdom and despite using multiple sites conclusions may not be able to be extrapolated to other countries with different health care systems.

\section{Recommendations}

The association of alcohol and drug use disorders with carer burden indicates the importance of screening and supporting caregivers of cancer patients. An individualised needs assessment is recommended to identify issues and implement support strategies. All patients started on strong painkillers should be monitored for drug misuse especially as many are now living with cancer, and taking painkillers, for longer time periods. 
Babor T, Higgins-Biddle J, Saunders J, Monteiro M. AUDIT: Guidelines for use in primary care. Second ed. Geneva: WHO; 2001.

Bayen E, Joudan C, Ghout I et al. Objective and subjective burden of informal caregivers 4 years after a severe traumatic brain injury: Results from the PariS-TBI Study. J Head Trauma Rehabil 2014 [Epub]

Bruera E, Moyano J, Seifert L, Fainsinger RL, Hanson J, Suarez-Almazor M. The frequency of alcoholism among patients with pain due to terminal cancer. J Pain Symptom Manage 1995;10:599-603.

Bruera E, Neumann C, Brenneis C, Quan H. Frequency of symptom distress and poor prognostic indicators in palliative cancer patients admitted to a tertiary palliative care unit, hospices, and acute care hospitals. J Palliat Care 2000;16:16-21.

Cancer Research UK (2016). Cancer Statistics for the UK. Cancer Research UK

Chang VT, Hwang SS, Feuerman M, Kasimis BS, Thaler HT. The memorial symptom assessment scale short form (MSAS-SF). Cancer 2000;89:1162-71.

Derogatis LR, Morrow GR, Fetting $\mathrm{J}$ et al. The prevalence of psychiatric disorders among cancer patients. JAMA 1983; 249: 751

Gallant MP, Connell CM. Predictors of decreased self-care among spouse caregivers of older adults with dementing illnesses. J Aging Health 1997; 9:373-95

Grov EK, Dahl AA, Moum T et al. Anxiety, depression and quality of life in caregivers of patients with cancer in late palliative phase. Ann Oncol 2005;1185-1191

Hotopf M, Chidgey J, Addington-Hall J et al. Depression in adavnced disease Part 1. Prevalence and case finding. Palliat Med 2002;16:81-97

Hsu T, Loscalzo M, Ramani R et al. Are disagreements in caregiver and patient assessment of patient health associated with increased caregiver burden in caregivers of older adults with cancer? Oncologist 2017; 22: 1383-1391.

Hsu T, Loscalzo M, Ramani R et al. Factors associated with high burden in caregivers of older adults with cancer. Cancer 2014; 120: 2927-35

Jenkins CA, Taube AW, Turner K, Hanson J, Bruera E. Initial demographic, symptom, and medication profiles in patients admitted to continuing palliative care units. J Pain Symptom Manage 1998;16:163-70.

Jenkins CA, Schulz M, Hanson J, Bruera E. Demographic, symptom, and medication profiles of cancer patients seen by a palliative care consult team in a tertiary referral hospital. J Pain Symptom Manage 2000;19:174-84.

Mayfield D, McLeod G, Hall P. The CAGE questionnaire: validation of a new alcoholism screening instrument. Am J Psychiatry 1974;131:1121-3. 
Mercadante S, Porzio G, Caruselli A et al. The frequency of alcoholism in patients with advanced cancer admitted to an acute palliative care unit and a home care program. $J$ Pain Symptom Manage. 2015; 45: 254-7

McManus S, Meltzer H, Brugha T, Bebbington P, Jenkins R. The NHS Information Centre for Health and Social Care. Adult Psychiatric Morbidity in England. London: The NHS Information Centre; 2007.

NHS Digital. Adult Psychiatric Morbidity Survey: Survey of Mental Health and Well-being, 2014. Office National Statistics. England

Office of National Statistics. Health survey for England 2017. NHS Digital: Government Statistical Service 2018

Parsons HA, Delgado-Guay MO, El Osta B, Chacko R, Poulter V, Palmer JL, et al. Alcoholism screening in patients with advanced cancer: impact on symptom burden and opioid use. J Palliat Med 2008;11:964-8.

Pinquart M, Sorenson S. Differences between caregivers and noncaregivers in psychological health and physical health: a meta-analysis. Psychol Aging 2003; 18: 250 267

Rumpold T, Schur S, Amering M et al. Informal caregivers of advanced-stage cancer patients: Every second is st risk for psychiatric morbidity. Support Care Cancer 2015 DOI $10.1007 / \mathrm{s} 00520-015-2987-z$

Schultz M, Boeing H, Pischon T et al. Alcohol attributable burden of incidence of cancer in eight European countries based on results from prospective cohort study. Br Med J 2011; 342: d1584

World Health Organisation: Expert committee on problems related to alcohol consumption. City: WHO; 2007.

Webber K, Davies AN. An observational study to determine the prevalence of alcohol use disorders in adavnced cancer patients. Palliat Med 2012; 26: 360-367

Webber K, Haber P. Alcohol and drug abuse in Palliative Medicine. In: Walsh TD, Caraceni AT, Fainsinger R, editors. Palliative Medicine: Expert consult premium edition. Elsevier Health Sciences; 2008

Yennurajalingam S, Edwards T, Arthur JA et al. Predicting the risk for aberrant opioid use behaviour in patients receiving outpatient supportive care consultation at a comprehensive cancer centre. Cancer 2018; 124: 3942-9

Webber K, Davies AN. Validity of the Memorial Symptom Assessment Scale-Short Form psychological subscales in advanced cancer patients. J Pain Symptom Manage 2011; 42: $761-767$

Yudko E, Lozhkina $O$ et al. A comprehensive review of the psychometric properties of the Drug Abuse Screening Test. J Subst Abuse Treat 2007; 32: 189-198

Zarit SH, Reever KE, Back-Peterson J. Relatives of the impaired elderly: correlates of feelings of burden. The Gerontologist 1980; 20: 649-55. 